\title{
Benefits of erectile function recovery programs after radical prostatectomy (Review)
}

\author{
DRAGOS RADU MARCU ${ }^{1,2}$, LUCIAN IORGA ${ }^{1}$, CAMELIA CRISTINA DIACONU ${ }^{2,3}$, \\ ARSENIE DAN SPINU ${ }^{1,2}$, DAN MISCHIANU ${ }^{1,2,4}$ and OVIDIU GABRIEL BRATU ${ }^{1,2,4}$ \\ ${ }^{1}$ Urology Clinic, 'Carol Davila' Central Military University Emergency Hospital, 010825 Bucharest; \\ ${ }^{2}$ Clinical Department 3, 'Carol Davila' University of Medicine and Pharmacy, 050474 Bucharest; ${ }^{3}$ Internal Medicine Clinic, \\ Clinical Emergency Hospital of Bucharest, 020322 Bucharest; ${ }^{4}$ Academy of Romanian Scientists, 030167 Bucharest, Romania
}

Received May 6, 2020; Accepted June 5, 2020

DOI: $10.3892 / \mathrm{etm} .2020 .8934$

\begin{abstract}
Radical prostatectomy is one of the most frequent therapeutic options used for the management of patients diagnosed with prostate cancer. Normal erectile function after radical prostatectomy is a great problem for numerous patients and a real challenge for urologists worldwide. The advancements that have been made over the years in terms of minimally invasive surgery, as well as in terms of surgical techniques, have reduced the incidence of erectile dysfunction, but even so, its rate remains high and the post-operative recovery of erectile function is a long and costly process. Phosphodiesterase 5 inhibitors have provided excellent results and have become the first-line treatment for these patients, followed by intracavernous injections with alprostadil. Several studies have underlined the impact of phosphodiesterase 5 inhibitors in terms of preventing the fibrotic changes that are responsible for the irreversible erectile dysfunction. The general opinion is that an erectile function recovery process should be started as soon as possible after surgery to prevent the negative effects of neuropraxia.
\end{abstract}

\section{Contents}

1. Introduction

2. Erectile dysfunction pathophysiology

3. The role of phosphodiesterase 5 -inhibitors in erectile function recovery

4. Discussion

Correspondence to: Dr Camelia Cristina Diaconu, Internal Medicine Clinic, Clinical Emergency Hospital of Bucharest, 8 Calea Floreasca, 020322 Bucharest, Romania

E-mail: drcameliadiaconu@gmail.com

Key words: erectile dysfunction, radical prostatectomy, PDE-5 inhibitors, intracavernous injections, alprostadil

\section{Introduction}

Prostate cancer (PCa) is one of the most frequent male malignancies worldwide. After the introduction of prostate-specific antigen (PSA) as a routine screening tool, it was noted that the incidence of advanced and metastatic PCa stages has constantly decreased, as increasing number of patients are diagnosed with localized PCa stages, which are potentially curable. This is in contrast to the pre-PSA era, when most patients were diagnosed in advanced stages, usually with metastatic disease, and associated poor prognosis (1).

Radical prostatectomy (RP) is one of the most frequent therapeutic options used for the management of patients diagnosed with $\mathrm{PCa}$. This procedure offers good results in terms of disease-free survival and overall survival, but like any other major surgical procedures, this also presents several possible disadvantages, such as postoperative erectile dysfunction (ED) and urinary incontinence. Because the mean age for the time of diagnosis has decreased, postoperative erectile function (EF) recovery represents an important issue among urologists $(2,3)$.

The preservation of the peri-prostatic neurovascular bundles has a major role in post-RP normal EF. The introduction of new surgical techniques that spare the neurovascular bundles has significantly improved post-RP EF. The criteria for such procedures are very clear, the European Association of Urology guidelines recommending that nerve-sparing should be performed only in patients diagnosed with low risk or intermediate risk localized PCa (4).

Although nerve-sparing (NS) techniques aim to preserve the EF, numerous patients complain of ED after surgery. Numerous factors can influence the baseline EF: Age, diabetes, obesity $(5,6)$, smoking, chronic alcohol abuse, depression, as well as antidepressants (7), opioids, ketoconazole and numerous other drugs (8), chronic kidney disease, neurologic diseases (Parkinson's disease, multiple sclerosis, myelodysplasia, tumors, epilepsy, peripheral neuropathy), hypogonadism, hyperprolactinemia, thyroid dysfunction (hyperthyroidism) and hypercortisolism (9-11).

Diabetes mellitus is one of the most frequent causes responsible for ED, these patients presenting a much higher risk of developing ED compared with non-diabetic patients (up to 
three times higher). Usually, diabetic patients develop ED with up to ten years earlier compared with the general population, this being the result of diabetes-associated vascular and peripheral nerve damage (12).

Cardiovascular disease is another important factor associated with ED. It is well known that ED is a sign of vascular disease and a predictor for ischemic cardiovascular events. Numerous studies report that ED precedes with up to two to three years a major cardiovascular event. The severity of ED correlates with the severity of vascular disease (coronary artery disease), as well as with the severity of cardiac symptoms. Therefore, the presence of ED should raise awareness over a possible silent cardiovascular disease (13).

Considering all these previously mentioned risk factors associated with ED, it is essential to perform a routine preoperative EF assessment, using validated questionnaires such as the International Index of Erectile Function (IIEF) questionnaire, to establish the baseline EF. This assessment is very important, because it can eliminate false expectations in terms of postoperative sexual function recovery (14).

\section{Erectile dysfunction pathophysiology}

Normal EF is a complex process that depends on intact neural signaling, normal vascular pathways and functional erectile tissue. The integrity of the cavernous nerves is essential for achieving a satisfactory penile tumescence because they release nitric oxide (NO) via nitric oxide synthase (NOS). The cavernous sinusoidal endothelial cells also produce NO. Once released, NO enhances the production of cyclic guanosine 3',5'-monophosphate (cGMP), which decreases the intracellular levels of calcium by increasing its efflux, thus favoring cavernous smooth muscle relaxation. Smooth muscle relaxation enhances the arterial blood flow and leads to engorgement of cavernous sinusoids. Once the cavernous sinusoids start to increase their volume, the pressure within the penis is raised and the venous plexus is compressed under the albuginea tunica, thus reducing the blood outflow and leading to penile tumescence $(10,15)$. Other important mediators that have an active role in the mechanism of penile erection are prostaglandin E1 (PGE1) and cyclic adenosine 3',5'-monophosphate (cAMP) (16).

ED following nerve-sparing RP is the result of neuropraxia. This phenomenon is unavoidable and it is the result of intraoperative pelvic and periprostatic tissue trauma, especially at the level of the neurovascular bundles, which leads to local inflammatory reactions and ischemia. Local hypoxia is associated with decreased PGE1 and cAMP levels, which alters the smooth muscle relaxation process at the level of cavernous cells, as well as with increased levels of profibrotic and proapoptotic factors, such endothelin 1 and transforming growth factor- $\beta$ (TGF- $\beta$ ) (15). Therefore, local hypoxia will lead to cavernosal structural changes, the smooth muscle fibers being gradually replaced by collagen and fibrotic tissue, which severely decreases the penile tumescence capability and also affects the vein-occlusive component of the erection, allowing blood outflow and affecting penile rigidity (17).

Several studies have investigated over the years the cavernous histological changes that appear after RP, as well as the hemodynamic changes. Mulhall et al (18) demonstrated that the vein-occlusive dysfunction is time-dependent, amplifying itself with the increasing local fibrosis.

The studies published by Nehra et al (19) in 1996, and by Iacono et al (20) in 2005 reported significant cavernous structural changes following RP, that consisted of the replacement of cavernous smooth muscle fibers by collagen tissue. The authors observed that the degree of vein-occlusive dysfunction was directly influenced by the balance between smooth muscle fibers and collagen tissue.

Considering all these previously described mechanisms involved in the pathophysiology of post RP ED, numerous attempts have been made over the years to prevent these irreversible changes that significantly alter the patients' well-being.

\section{The role of phosphodiesterase 5-inhibitors in EF recovery}

The introduction of phosphodiesterase 5-inhibitors (PDE5-i) in the late 90 's has changed the management of ED, these drugs proving to be an excellent option for patients suffering from ED. Numerous studies have evaluated the efficiency of PDE5-i in terms of post radical prostatectomy ED management (21).

The Reinvent clinical trial evaluated the efficiency of daily versus on-demand vardenafil. Over 600 patients who had undergone NSRP were randomized into three groups: group 1, daily $10 \mathrm{mg}$ vardenafil and placebo on-demand; group 2, daily placebo plus on-demand vardenafil; and group 3, daily placebo and on-demand placebo. This trial had three phases: 9 months double-blind treatment, followed by a two months washout period and afterward another two months of open-label vardenafil. At the end of the study, the authors concluded that nightly vardenafil did not prove to be superior to on-demand vardenafil (22).

A similar conclusion was published by Pavlovich et al (23) in 2013. The patients enrolled in this trial have received either nightly sildenafil plus on-demand placebo or on-demand sildenafil plus daily placebo for one year. Daily sildenafil did not offer superior results compared with on-demand sildenafil, underlining that its chronic daily use is not justifiable for EF recovery in patients who have undergone bilateral NSRP.

Another study that was conducted over 12 months, followed by 1 month washout period, has aimed at identifying whether sildenafil administered daily is superior to on-demand sildenafil. At the end of this trial, the daily usage of sildenafil did not reach superior results compared with the on-demand sildenafil group (24).

The REACT clinical trial evaluated nightly versus on-demand tadalafil. The patients were randomized into three groups: $5 \mathrm{mg}$ daily tadalafil, $20 \mathrm{mg}$ on-demand tadalafil, and placebo. The patients received treatment for nine months, followed by a washout period of 6 weeks and afterward by a period of three months of daily tadalafil. The results achieved at the end of the first nine months have shown that daily tadalafil was superior to placebo, as well as to on-demand tadalafil. Regarding the efficiency of on-demand tadalafil versus placebo, the results did not reach a statistical significance. At the end of the drug washout period, the superiority of daily tadalafil was not maintained. After reintroducing daily tadalafil for three months, the IIEF and Sexual Encounter Profile-3 (SEP3) scores improved. In terms of penile shrinkage prevention, the daily usage of tadalafil has demonstrated superior results (25). 
Kim and Sung (26) compared whether daily tadalafil administered for one year is inferior or not to tadalafil administered daily for two years. The study included 95 patients who had undergone NSRP. No staistically significant difference was noted at the end of the study between the two tadalafil groups (two years versus one year) in terms of EF recovery. The authors also reported that they did not reach any major statistical differences when they compared the patients with bilateral NSRP and those for whom unilateral NSRP was performed.

In a randomized clinical trial, Moncada et al (27) concluded that daily administration of tadalafil speeds up the EF recovery process. Their study included 423 patients who had undergone bilateral NSRP. The patients were randomized to receive either daily $5 \mathrm{mg}$ tadalafil or on-demand $20 \mathrm{mg}$ tadalafil or placebo, for nine months. The study also included a 6-week drug washout period and another twelve weeks of daily tadalafil for all the three groups of patients. The authors stated that the time of the treatment was too short for $>50 \%$ of the patients to achieve a satisfactory erection recovery. They further reported that $25 \%$ of the patients reached an EF recovery (IIEF-EF $\geq 22$ ) over a period that ranged between 5.8 and 9.3 months (5.8 months for patients with daily tadalafil versus 9 months for those with on-demand tadalfil and 9.3 months for patients who have received placebo), the daily administration of tadalafil favoring a faster EF recovery.

\section{Discussion}

Surgical experience and surgical approach are key elements that influence the postoperative EF recovery. Potdevin et al (28) compared the intra-fascial nerve-sparing technique with the inter-fascial technique and reported that the intra-fascial nerve-sparing approach offers superior results in terms of $\mathrm{EF}$ recovery. Numerous studies have reported the superiority of the robot-assisted approach over open and laparoscopic RP (ORP and LRP) in terms of nerve-sparing success and EF recovery.

Stolzenburg et al (2) analyzed the REACTT clinical trial in terms of surgical approach and its influence over EF impairment and reported that patients who had undergone nerve-sparing RARP associated superior chances (twice as high) of recovering a satisfactory EF (IIEF-EF $\geq 22$ ) at the end of the drug washout period, as well as at the end of the open-label tadalafil period, compared with those for whom ORP or LRP was performed. When comparing the superiority of LRP over ORP in terms of postoperative erectile function recovery, no significant statistical difference has been achieved.

A study published in 2011, conducted on 128 patients who have undergone either RARP or LRP, concluded that the robotic approach associated with higher IIEF scores twelve months following surgery, as well as increased rates of EF recovery similar to baseline $\mathrm{EF}$ and a significantly faster $\mathrm{EF}$ recovery (29).

Greco et al (30) analyzed 457 patients who had undergone nerve-sparing LRP. The authors reported that EF assessed at twelve months following surgery was superior in patients for whom bilateral NS was achieved compared with those with unilateral NS (69 vs. 43\%). On-demand $20 \mathrm{mg}$ vardenafil was used after surgery, in order to improve the EF.
Another viable solution that could be used for postoperative EF recovery is represented by intracavernous injections (ICI) with alprostadil. The first mention of an EF recovery program following RP was made by Montorsi et al (31) in a study published in 1997 and it implied alprostadil ICI. This trial included 30 patients who had undergone bilateral NSRP. Following surgery, the patients were randomized to receive ICI with alprostadil three times a week, for twelve weeks, or no treatment. The authors reported that patients included in the ICI group achieved superior results in terms of EF recovery compared with the observational group (67 vs. $20 \%$ ).

Numerous other studies underline the positive effect of intra-cavernous injections with alprostadil, but their use is much more limited compared with PDE5-inhibitors. This is usually related to the fact that patients must self-administer the injections and also to the discomfort/pain associated with these injections (32-35).

A study in 2015 (36) aimed at finding out if a longer alprostadil protocol could improve the postoperative erectile function in terms of spontaneous nocturnal erections. Two times a week ICIs with alprostadil were recommended, starting the first month following surgery. At approximately one year after the initiation of the alprostadil treatment, the patients were recommended to use PDE5-inhibitors and the non-responders were encouraged to continue the ICIs for another 12 months. The authors reported that alprostadil had significantly improved the patients' quality of life, but the results in terms of spontaneous nocturnal erections, between one year and two years of follow-up, were weak. The satisfaction of patients regarding the treatment efficiency had shown a decline between the 12th and 24th month.

The benefits of an EF recovery program have been underlined in numerous studies, but a clear protocol is lacking, regarding the time of treatment initiation, drug choice, administration frequency, and treatment length. According to literature, PDE5-i are the most common choice among urologists, due to their ease of use and safety, as well as their good results. The time of PDE5-i treatment initiation is not very clear, numerous studies reporting their administration several weeks after surgery, and other studies report earlier use, such as the next day following surgery or even before surgery. Another important aspect is whether chronic daily administration of PDE5 inhibitors is better than on-demand use, because the information available on this subject is, to a certain extent, contradictory. Numerous studies report the advantages of nightly administration over on-demand use, but several clinical trials have failed to prove it. In conclusion, ED after RP is an important health problem, especially because the mean age of patients diagnosed with $\mathrm{PCa}$ has decreased, due to the use of PSA and multiparametric MRI. Numerous studies have underlined the role of EF recovering programs, but a clear protocol, in terms of drug choice, treatment initiation and its extent, still does not exist. Nevertheless, PDE5-i have proven to be a good option for such patients. Their good results and ease of use have made them the first-line treatment for post-RP ED. Despite conflicting information whether daily usage is superior to on-demand administration, the majority of the existing studies favor their daily administration, as well as an early treatment initiation. Intra-cavernous alprostadil injections also provide good results, but due to their associated disadvantages, they remain a second option. 


\section{Acknowledgements}

Not applicable.

\section{Funding}

No funding was received.

\section{Availability of data and materials}

Not applicable.

\section{Authors' contribution}

DRM, LI, CCD and ADS collected, analyzed and interpreted the patient data regarding the erectile function recovery after radical prostatectomy. CCD, OGB and DM had substantial contribution to the conception of the study and interpretation of the data. DRM, CCD and OGB drafted the manuscript and were major contributors in the writing of the manuscript. All authors read and approved the final version of the manuscript.

\section{Ethics approval and consent to participate}

Not applicable.

\section{Patient consent for publication}

Not applicable.

\section{Competing interests}

The authors declare that they have no competing interests.

\section{References}

1. Rao AR, Motiwala HG and Karim OM: The discovery of prostate-specific antigen. BJU Int 101: 5-10, 2008.

2. Stolzenburg JU, Graefen M, Kriegel C, Michl U, Martin Morales A, Pommerville PJ, Manning M, Büttner H, Henneges C and Schostak M: Effect of surgical approach on erectile function recovery following bilateral nerve-sparing radical prostatectomy: An evaluation utilising data from a randomised, double-blind, double-dummy multicentre trial of tadalafil vs. placebo. BJU Int 116: 241-251, 2015.

3. Bratu OG, Diaconu CC, Mischianu DL, Constantin T, Stanescu AM, Bungau SG, Ionita-Radu F and Marcu RD: Therapeutic options in patients with biochemical recurrence after radical prostatectomy (Review). Exp Ther Med 18: 5021-5025, 2019.

4. European Association of Urology: Prostate cancer guidelines. https://uroweb.org/guideline/prostate-cancer/\#6. Accessed Jan 20, 2020.

5. Vesa CM,Popa AR, Bungau S, Daina LG, Buhas C, Judea-Pusta CT and Pasca B, Dimulescu IA and Zaha DC: Exploration of insulin sensitivity, insulin resistance, early insulin secretion and $\beta$-cell function, and their relationship with glycated hemoglobin level in normal weight patients with newly diagnosed type 2 diabetes mellitus. Rev Chim 70: 4217-4223, 2019.

6. Popa AR, Bungau S, Vesa CM, Bondar AC, Pantis C and Maghiar O, Dimulescu IA, Nistor-Cseppento DC and Rus M: Evaluating the efficacy of the treatment with benfotiamine and alpha-lipoic acid in distal symmetric painful diabetic polyneuropathy. Rev Chim 70: 3108-3114, 2019.

7. Purza L, Abdel-Daim M, Belba A, Iovan C, Bumbu A, Lazar L, Bungau $S$ and Tit DM: Monitoring the effects of various combination of specific drug therapies at different stages of Alzheimer's dementia. Farmacia 67: 477-481, 2019.
8. Zaha DC, Bungau S, Aleya S, Tit DM, Vesa CM, Popa AR, Pantis C, Maghiar OA, Bratu OG, Furau C, et al: What antibiotics for what pathogens? The sensitivity spectrum of isolated strains in an intensive care unit. Sci Total Environ 687: 118-127, 2019.

9. Kadıŏlu A, Ortaç M, Dinçer M and Brock G: Tadalafil therapy for erectile dysfunction following prostatectomy. Ther Adv Urol 7: 146-151, 2015.

10. Barazani Y, Stahl PJ, Nagler HM and Stember DS: Is there a rationale for penile rehabilitation following radical prostatectomy? Am J Men Health 9: 35-43, 2015.

11. Abdel-Daim MM, El-Tawil OS, Bungau SG and Atanasov AG: Applications of antioxidants in metabolic disorders and degenerative siseases: Mechanistic approach. Oxid Med Cell Longev 2019: 4179676, 2019.

12. Anwar Z, Sinha V, Mitra S, Mishra AK, Ansari MH, Bharti A, Kumar V and Nigam AK: Erectile dysfunction: An underestimated presentation in patients with diabetes mellitus. Indian J Psychol Med 39: 600-604, 2017.

13. Montorsi P, Ravagnani PM, Galli S, Rotatori F, Veglia F, Briganti A, Salonia A, Dehò F, Rigatti P, Montorsi F, et al: Association between erectile dysfunction and coronary artery disease. Role of coronary clinical presentation and extent of coronary vessels involvement: The COBRA trial. Eur Heart J 27: 2632-2639, 2006.

14. Gandaglia G, Suardi N, Cucchiara V, Bianchi M, Shariat SF, Roupret M, Salonia A, Montorsi F and Briganti A: Penile rehabilitation after radical prostatectomy: Does it work? Transl Androl Urol 4: 110-123, 2015.

15. Blecher G, Almekaty K, Kalejaiye O and Minhas S: Does penile rehabilitation have a role in the treatment of erectile dysfunction following radical prostatectomy? F1000Res 6: 1923, 2017.

16. Ben Khedher MR, Bouhajja H, Haj Ahmed S, Abid M, Jamoussi K and Hammami M: Role of disturbed fatty acids metabolism in the pathophysiology of diabetic erectile dysfunction. Lipids Health Dis 16: 241, 2017.

17. Mulhall JP: Penile rehabilitation following radical prostatectomy. Curr Opin Urol 18: 613-620, 2008.

18. Mulhall JP, Slovick R, Hotaling J, Aviv N, Valenzuela R, Waters WB and Flanigan RC: Erectile dysfunction after radical prostatectomy: Hemodynamic profiles and their correlation with the recovery of erectile function. J Urol 167: 1371-1375, 2002.

19. Nehra A, Goldstein I, Pabby A, Nugent M, Huang YH, de las Morenas A, Krane RJ, Udelson D, Saenz de Tejada I and Moreland RB: Mechanisms of venous leakage: A prospective clinicopathological correlation of corporeal function and structure. J Urol 156: 1320-1329, 1996.

20. Iacono F, Giannella R, Somma P, Manno G, Fusco F and Mirone V: Histological alterations in cavernous tissue after radical prostatectomy. J Urol 173: 1673-1676, 2005.

21. Clavell-Hernandez J and Wang R: Penile rehabilitation following prostate cancer treatment: Review of current literature. Asian J Androl 17: 916-922, 2015.

22. Montorsi F, Brock G, Lee J, Shapiro J, Van Poppel H, Graefen M and Stief C: Effect of nightly versus on-demand vardenafil on recovery of erectile function in men following bilateral nerve-sparing radical prostatectomy. Eur Urol 54: 924-931, 2008.

23. Pavlovich CP, Levinson AW, Su LM, Mettee LZ, Feng Z, Bivalacqua TJ and Trock BJ: Nightly vs on-demand sildenafil for penile rehabilitation after minimally invasive nerve-sparing radical prostatectomy: Results of a randomized double-blind trial with placebo. BJU Int 112: 844-851, 2013.

24. Kim DJ,Hawksworth DJ, Hurwitz LM, Cullen J, Rosner IL, Lue TF and Dean RC: A prospective, randomized, placebo-controlled trial of on-Demand vs. nightly sildenafil citrate as assessed by Rigiscan and the international index of erectile function. Andrology 4: 27-32, 2016.

25. Montorsi F, Brock G, Stolzenburg JU, Mulhall J, Moncada I, Patel HR, Chevallier D, Krajka K, Henneges C, Dickson R, et al: Effects of tadalafil treatment on erectile function recovery following bilateral nerve-sparing radical prostatectomy: A randomised placebo-controlled study (REACTT). Eur Urol 65: 587-596, 2014.

26. Kim S and Sung GT: Efficacy and safety of tadalafil $5 \mathrm{mg}$ once daily for the treatment of erectile dysfunction after robot-assisted laparoscopic radical prostatectomy: A 2-year follow-up. Sex Med 6: 108-114, 2018.

27. Moncada I, de Bethencourt FR, Lledó-García E, Romero-Otero J, Turbi C, Büttner H, Henneges C and Martinez Salamanca JI: Effects of tadalafil once daily or on demand versus placebo on time to recovery of erectile function in patients after bilateral nerve-sparing radical prostatectomy. World J Urol 33: 1031-1038, 2015. 
28. Potdevin L, Ercolani M, Jeong J and Kim IY: Functional and oncologic outcomes comparing interfascial and intrafascial nerve sparing in robot-assisted laparoscopic radical prostatectomies. J Endourol 23: 1479-1484, 2009.

29. Asimakopoulos AD, Pereira Fraga CT, Annino F, Pasqualetti P, Calado AA and Mugnier C: Randomized comparison between laparoscopic and robot-assisted nerve-sparing radical prostatectomy. J Sex Med 8: 1503-1512, 2011.

30. Greco F, Hoda MR, Wagner S, Reichelt O, Inferrera A, Magno C and Fornara P: Bilateral vs. unilateral laparoscopic intrafascial nerve-sparing radical prostatectomy: Evaluation of surgical and functional outcomes in 457 patients. BJU Int 108: 583-587, 2011.

31. Montorsi F, Guazzoni G, Strambi LF, Da Pozzo LF, Nava L, Barbieri L, Rigatti P, Pizzini G and Miani A: Recovery of spontaneous erectile function after nerve-sparing radical retropubic prostatectomy with and without early intracavernous injections of alprostadil: Results of a prospective, randomized trial. J Urol 158: 1408-1410, 1997.

32. Bratu O, Spinu D, Oprea I, Popescu R, Marcu D, Farcas C, Dinu $M$ and Mischianu D: Complications of radical retropubic prostatectomy - our experience. Rom J Mil Med 118: 23-25, 2015.
33. Marcu D, Spinu D, Mischianu D, Socea B, Oprea I and Bratu O: Oncological follow-up after radical prostatectomy. Rom J Mil Med 120: 39-42, 2017.

34. Popescu R, Bratu O, Spinu D, Marcu D, Farcas C, Dinu M and Mischianu D: Neuroendocrine differentiation in prostate cancer - a review. Rom J Mil Med 118: 16-19, 2015.

35. Cherciu A, Spinu D, Sandru F, Marcu D, Iorga L, Anghe R, Bratu $\mathrm{O}$ and Mischianu D: Erectile disfunction after radical prostatectomy. Ann Acad Rom Scientists Ser Med 1: 29-34, 2020.

36. Yiou R, Bütow Z, Parisot J, Binhas M, Lingombet O, Augustin D, de la Taille A and Audureau E: Is it worth continuing sexual rehabilitation after radical prostatectomy with intracavernous injection of alprostadil for more than 1 year? Sex Med 3: 42-48, 2015. 